\title{
LEVANTAMENTO DAS FAMÍLIAS DE HIMENÓPTEROS PARASITOIDES ASSOCIADOS AO CULTIVO DE TABACO (NICOTIANA TABACUM L.) EM SANTA CRUZ DO SUL E LAGOÃO,RIO GRANDE DO SUL, BRASIL
}

\author{
C. Dorfey, K. Schoeninger, A. Köhler \\ Universidade de Santa Cruz do Sul, Laboratório de Entomologia, Av. Independência, 2293, CEP 96815-900, \\ Santa Cruz do Sul, RS, Brasil.
}

\section{RESUMO}

\begin{abstract}
O objetivo deste trabalho foi realizar o levantamento das famílias de himenópteros parasitoides associados ao cultivo do tabaco (Nicotiana tabacum L) no Estado do Rio Grande do Sul. Foram realizadas coletas quinzenais com armadilhas de Malaise, pitfalls e bandejas amarelas em lavouras de tabaco nos municípios de Santa Cruz do Sul e Lagoão. Durante o período de novembro de 2007 a fevereiro de 2008, foram coletados 1.047 indivíduos da ordem Hymenoptera. Destes, 738 parasitoides estão distribuídos em 23 famílias. Ichneumonidae, Braconidae e Scelionidae foram as famílias mais abundantes, representando 44,6\%, 14,9\% e 11,5\% dos indivíduos parasitoides coletados, respectivamente.
\end{abstract}

PALAVRAS-CHAVE: Entomofauna, biodiversidade, Hymenoptera, parasitoide, tabaco orgânico.

\section{ABSTRACT}

SURVEY OF THE FAMILIES OF PARASITIC HYMENOPTERA ASSOCIATED WITH TOBACCO (NICOTIANA TABACUM L.) CROPS IN RIO GRANDE DO SUL STATE, BRAZIL. The aim of this work was to survey the families of hymenoptera associated with tobacco (Nicotiana tabacum L.) in Rio Grande do Sul State, Brazil. Material was collected weekly using Malaise, pitfall and yellow pan traps in tobacco crops in the counties of Santa Cruz do Sul and Lagoão. During the period of November 2007 to February 2008, 1,047 individuals belonging to the order Hymenoptera were collected. Of these, 738 were distributed in 23 families known as parasitoid. Ichneumonidae, Braconidae and Scelionidae were the most abundant families, representing $44.6 \%, 14.9 \%$ and $11.5 \%$ of the collected parasitoids, respectively.

KEY WORDS: Entomofauna, biodiversity, Hymenoptera, parasitoid, organic tobacco.

Dentre as espécies de Hymenoptera, vários insetos de hábito predador ou parasitoide têm sido relatados como inimigos naturais de insetos-praga (PERIOTO et al., 2004). Os himenópteros parasitoides são um importante elemento da fauna neotropical por seu papel no controle da população de outros insetos que interferem, direta ou indiretamente e de forma ainda não bem quantificada, nas cadeias tróficas de grande parte dos agroecossistemas (РЕRIOTO et al., 2004).

A fauna de himenópteros parasitoides é pouco conhecida no Brasil, apesar de sua grande diversidadeeimportância biológica, ecológica eeconômica. SegundoSouzAet al. (2006), a literatura sobre os himenópteros parasitoides aborda principalmente a taxonomia, a biologia e o uso em controle biológico, mas há poucos trabalhos sobre a composição faunística.

Com relação ao cultivo do tabaco (Nicotiana tabacum L.), não existem trabalhos quantitativos e qualitativose/ou levantamentos publicados sobrea diversidade de parasitoides, demonstrando a necessidade de inventariamento dos inimigos naturais que ocorrem nessa cultura e realizam ocontrolebiológico natural das pragas.

O objetivo deste trabalho foi realizar o levantamento das famílias dehimenópteros parasitoides associadas ao cultivo do tabaco nos municípios deSanta Cruz doSul e Lagoão, RS, Brasil. Para tanto, em duas lavouras de tabaco (uma no Município de Lagoão e outra localizada em Santa Cruz do Sul), foram instaladas 2 armadilhas de Malaise (para captura de insetos em atividade de vôo), 4 armadilhas tipo pitfall (para captura de insetos epígeos) e 4 bandejas amarelas (atração visual). As coletas foram realizadas quinzenalmente no período de novembro de 2007 a fevereiro de 2008, sendo os indivíduos coletados identificados em nível de família segundo Goulet; Huber (1993), fixados em álcool 70\% e depositados na Coleção Entomológica da Universidade de Santa Cruz do Sul (CESC). 
Foram coletados um total de 1.047 espécimes pertencentes à ordem Hymenoptera, distribuídos em 36 famílias. Destes indivíduos, 738 pertencem às 23 famílias consideradas parasitoides segundo a bibliografia mundial (Tabela 1).
Aocorrênciade23famíliasparasitoidesemcultivode tabacodemonstraalta diversidade. NoBrasilocorrem 37 famíliaseafaunamundialabrange 61 famílias, sendoque várias destas são exclusivas de regiões zoogeográficas como a Australiana e a Holártica (AzEvedo et al., 2002).

Tabela 1 - Himenópteros parasitoides coletados no período de novembro de 2007 a fevereiro de 2008 associados a plantas de tabaco (N. tabacum) nos municípios de Santa Cruz do Sul e Lagoão, RS, Brasil.

\begin{tabular}{|c|c|c|c|}
\hline Superfamília & Família & Quantidade & Frequência (\%) \\
\hline \multicolumn{4}{|c|}{ Ceraphronoidea } \\
\hline & Ceraphronidae & 2 & 0,27 \\
\hline & Megaspilidae & 2 & 0,27 \\
\hline \multicolumn{4}{|c|}{ Chalcidoidea } \\
\hline & Chalcididae & 29 & 3,93 \\
\hline & Encyrtidae & 3 & 0,41 \\
\hline & Eulophidae & 2 & 0,27 \\
\hline & Eupelmidae & 2 & 0,27 \\
\hline & Eurytomidae & 2 & 0,27 \\
\hline & Mymaridae & 9 & 1,22 \\
\hline & Perilampidae & 1 & 0,13 \\
\hline & Pteromalidae & 1 & 0,13 \\
\hline & Tanaostigmatidae & 1 & 0,13 \\
\hline & Torymidae & 1 & 0,13 \\
\hline \multicolumn{4}{|c|}{ Chrysidoidea } \\
\hline & Bethylidae & 67 & 9,10 \\
\hline & Chrysididae & 14 & 1,9 \\
\hline \multicolumn{4}{|l|}{ Cynipoidea } \\
\hline & Figitidae & 4 & 0,54 \\
\hline \multicolumn{4}{|l|}{ Evanioidea } \\
\hline & Aulacidae & 6 & 0,81 \\
\hline & Evaniidae & 16 & 2,17 \\
\hline \multicolumn{4}{|c|}{ Ichneumonoidea } \\
\hline & Braconidae & 110 & 14,91 \\
\hline & Ichneumonidae & 329 & 44,58 \\
\hline \multicolumn{4}{|c|}{ Platygastroidea } \\
\hline & Scelionidae & 85 & 11,52 \\
\hline \multicolumn{4}{|c|}{ Proctotrupoidea } \\
\hline & Diapriidae & 2 & 0,27 \\
\hline \multicolumn{4}{|l|}{ Vespoidea } \\
\hline & Pompilidae & 46 & 6,23 \\
\hline & Tiphiidae & 4 & 0,54 \\
\hline Total & & 738 & 100 \\
\hline
\end{tabular}


As famílias Ichneumonidae e Braconidae foram as mais representativas neste trabalho, correspondendo a $59,49 \%$ dos indivíduos parasitoides coletados. Ambas as famílias pertencem à Ichneumonoidea, a qual representa um dos principais táxons, tanto em número como em eficiência parasitária, podendo atacar ovos, larvas, pupas ou imagos de outros insetos (SCatolini; Penteado-Dias, 1997). AzEVEDo et al. (2003) destacaram a ocorrência de Ichneumonidae e Braconidae em área de Mata Atlântica na Estação Biológica de Santa Lúcia, Santa Teresa, ES, assim como Amaral et al. (2006) em Luz, MG, onde Ichneumonidae representou $42 \%$ do total coletado com armadilha de Malaise. No entanto, quando associados a cultivos agrícolas, a incidência destas famílias é baixa, conforme Perioto et al. (2002a) que verificaram a baixa ocorrência da superfamília Ichneumonoidea em cultura de algodão, representando $2,56 \%$ do total de himenópteros parasitoides coletados; e SouzA et al. (2006) que registraram pouca representatividade de Ichneumonoidea em área agrícola do Município de Rio Claro, SP, correspondendo a 4,09\%. Os autores citam o fato de que a ocorrência de Ichneumonidae pode estar relacionada com umidade e, portanto, a frequência encontrada naquele estudo pode refletir a pressão ambiental exercida sobre o grupo em função da dinâmica do plantio naquela área agrícola. No presente trabalho, a alta incidência de Ichneumonidae e Braconidae demonstra que a ocorrência de espécies dessas famílias em cultivo de tabaco pode ser atribuída à existência de um grande número de hospedeiros potenciais das mesmas na cultura em questão.

Scelionidae foi a terceira família de parasitoides mais frequente associada ao tabaco, correspondendo a 11,52\% dos indivíduos coletados. Perioto et al. (2002a) e Perioto et al. (2002b) destacaram a ocorrência de Scelionidae em cultivos de soja e algodão no Estado de São Paulo. No mesmo Estado, Souza et al.(2006) destacaram a ocorrência desta família em área agrícola, onde foram cultivados sorgo, milho, feijão e trigo, em sistema de rodízio, em plantio direto. No Estado do Rio Grande do Sul, SANTos et al. (2001) registraram a ocorrência da espécie Gryon gallardoi (Brèthes) (Hym.: Scelionidae) como parasitoide de Spartocera dentiventris (Berg) (Hem.: Coreidae) em cultivo de tabaco.

As famílias Bethylidae, Pompilidae e Chalcididae representaram $9,08 \%, 6,23 \%$ e $3,93 \%$ dos indivíduos parasitoides coletados, respectivamente. As demais famílias apresentaram frequência abaixo de 3\%.

Os dados obtidos demonstram a diversidade quanto à disponibilidade de inimigos naturais associados ao cultivo do tabaco na região produtora de Santa Cruz do Sul, constituindo um dos primeiros trabalhos a inventariar a entomofauna de parasitoides - qualitativamente e quantitativamente - nesta cultura. Verifica-se a necessidade de estudos mais específicos sobre a taxonomia de himenópteros parasitoides e seus possíveis usos em programas de controle biológico e/ou manejo integrado de pragas em cultivo de tabaco.

\section{REFERÊNCIAS}

AMARAL, D.P.; FONSECA, A.R.; SILVA, C. G.; SILVA, F.M.; ALVARENGA JÚNIOR, A. Diversidade de famílias de parasitóides (Hymenoptera: Insecta) coletados com armadilhas Malaise em floresta nativa em Luz, Estados de Minas Gerais, Brasil. Arquivos do Instituto Biológico, São Paulo, v.72, n.4, p.543-545, 2005.

AZEVEDO, C.O.; KAWADA, R.; TAVARES, M.T.; PERIOTO, N.W. Perfil da fauna de himenópteros parasitóides (Insecta, Hymenoptera) em uma área de Mata Atlantica do Parque Estadual da Fonte Grande, Vitoria, ES, Brasil. Revista Brasileira de Entomologia, v.46, n.2, p.133-137, 2002.

AZEVEDO, C.O.; CORREA, M.A.; GOBBI, F.T.; KAWADA, R.; LANES, G.O.; MOREIRA, A.R.; REDIGHIERI, E.S.; SANTOS, L.M.; WAICHERT, C. Perfil das famílias de vespas parasitóides (Hymenoptera) em uma área de Mata Atlântica da Estação Biológica de Santa Lúcia, Santa Teresa, ES, Brasil. Boletim do Museu Biológico Mello Leitão, n.16, p.39-46, 2003.

GOULET, H.; HUBER, J.T. Hymenoptera of the World: An identification guide to families. Ottawa: Agriculture Canada, 1993.

PERIOTO, N.W.; LARA, R.I.R.; SANTOS, J.C.C.; SELEGATTO, A. Himenópteros parasitóides (Insecta: Hymenoptera) coletados em cultura de algodão (Gossypium hirsutum L.) (Malvaceae), no município de Ribeirão Preto, SP, Brasil. Revista Brasileira de Entomologia, v.46, n.2, p.165-168, 2002a.

PERIOTO, N.W.; LARA, R.I.R.; SANTOS, J.C.C.; SILVA, T.C. Himenópteros parasitóides (Insecta, Hymenoptera) coletados em cultura de soja (Glycine max (L.)) Merril (Fabaceae), no município de Nuporanga, SP, Brasil. Revista Brasileira de Entomologia, v.46, n.2, p.185-187, 2002 b.

PERIOTO, N.W.; LARA, R.I.R.; SELEGATTO, A.; LUCIANO, E.S. Himenópteros parasitóides (Insecta: Hymenoptera) coletados em cultura de café Coffea arabica L. (Rubiaceae) em Ribeirão Preto, SP, Brasil. Arquivos do Instituto Biológico, São Paulo, v.71, n.1, p.41-44, 2004.

SANTOS, R.S.S., REDAELLI, L.R.; DIEFENBACH, L.M.G. Ocorrência de parasitismo em ovos de Spartocera dentiventris (Berg) (Hemiptera: Coreidae) em cultura de fumo. Neotropical Entomology, v.30, n.4, p.731-733, 2001.

SCATOLINI, D.; PENTEADO-DIAS, A.M. Fauna de Braconidae (Hymenoptera) como bioindicadora do grau de preservação de duas localidades do Estado do Paraná. Revista Brasileira Ecologia, v.1, n.1, p. 84-87, 1997.

SOUZA, L.; BRAGA, S.M.P.; CAMPOS, M.J.O. Himenópteros parasitóides (Insecta, Hymenoptera) coletados em área agrícola de Rio Claro, SP, Brasil. Arquivos do Instituto Biológico, São Paulo, v.73, n.4, p.465-469, 2006.

Recebido em 26/10/09

Aceito em 20/5/11 